hep-th/9911137

TIFR-TH/99-57

MRI-PHY/P99113

$\operatorname{IMSc} / 11 / 37$

\title{
Supergravity with Self-dual $B$ fields and Instantons in Noncommutative Gauge Theory
}

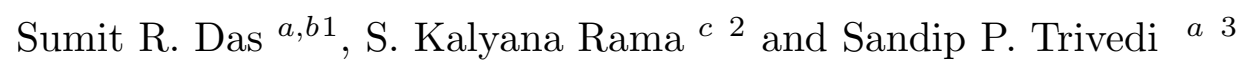 \\ a Tata Institute of Fundamental Research \\ Homi Bhabha Road, Bombay 400 005, INDIA \\ ${ }^{b}$ Mehta Research Institute of Mathematics and Mathematical Physics \\ Jhusi, Allahabad 211 019, INDIA \\ ${ }^{c}$ Institute of Mathematical Sciences \\ C.I.T. Campus, Chennai 600113, INDIA
}

\begin{abstract}
We study Type IIB supergravity in the presence of (euclidean) D3 branes and nonzero self-dual $B$-fields. We point out that the Einstein frame metric is identical to the full geometry for D3 branes without B fields turned on. Furthermore, in a decoupling limit in which the theory is conjectured to be dual to noncommutative Yang-Mills theory, the entire Einstein metric remains intact, and in particular, is asymptotically flat. We construct D-instanton solutions in this geometry. We show that in the decoupling limit the D-instanton action agrees with the action of the corresponding instanton in the noncommutative Yang-Mills theory and is expressed in terms of the open string coupling. Some other aspects of this correspondence, which have unusual features because the underlying metric is asymptotically flat, are explored.
\end{abstract}

November, 1999

\footnotetext{
1 das@theory.tifr.res.in

2 krama@imsc.ernet.in

3 sandip@theory.tifr.res.in
} 


\section{Introduction}

The observation that the low energy dynamics of D-branes in the presence of constant NSNS B fields is noncommutative Yang-Mills theory [1] has opened up the possibility of exploring string theory in a new limit. In this limit the string tension is scaled to infinity, and at the same time the closed string metric is scaled to zero, keeping the dimensionful NSNS B field fixed [2]. This is different from the usual low energy limit where the closed string metric and the dimensionless NSNS B-field are kept fixed as the tension scales to infinity. As a result, the lowest order effective action on the brane contains an infinite number of terms with all powers of the field strength when written in terms of ordinary gauge fields and conventional gauge symmetry. However, as shown in [2], in terms of noncommutative gauge fields, one has a simple lagrangian. The fact that an infinite number of terms may be neatly summarized in terms of a different geometric structure raises hopes that one may be able to perform concrete calculations in this new corner of string theory. Various aspects of noncommutative gauge theory and its relationship with string theory have been studied in [3].

Over the past two years it has become clear that large-N supersymmetric Yang-Mills theories are holographic descriptions of string theories in suitable backgrounds, [4] one of the best understood example being the duality between large- $\mathrm{N} \mathcal{N}=4$ Yang-Mills theory in four space-time dimensions and $I I B$ string theory on $A d S_{5} \times S^{5}$. When the 't Hooft coupling of the Yang-Mills theory is strong the dual string theory reduces to supergravity. The Yang-Mills theory in question is the low energy theory of $N$ three branes and the $A d S_{5} \times S^{5}$ background of the supergravity is the near-horizon limit of the three-brane background. It is natural to expect that in the presence of a $B$ field, one would have a similar correspondence between noncomuutative Yang-Mills theory and supergravity backgrounds in the presence of suitable $B$ fields. Indeed in [5] and [6] dual supergravity backgrounds for noncommutative boundary theories were proposed and certain aspects of holography were investigated in [6] and [7]. Other examples of such backgrounds have recently appeared in $[8]$.

In this paper we will continue the study of the supergravity background dual to $3+1$ dimensional noncommutative $S U(N)$ Yang Mills theory. There are two motivations for this study. First, as in the ordinary case, one might hope to learn something about noncommutative Yang Mills theory at large 'tHooft coupling in this way. Second, and perhaps more interestingly, one might hope to learn about holography in contexts other than those of AdS space. 
In particular we will focus on the case where the two form $B$ fields are self dual. In this case as was discussed in [2] the instanton moduli space in the noncommutative Yang Mills theory is the same as that of ordinary Yang Mills theory and in particular, the size of the instanton is one of the moduli. Correspondingly, the D-instanton is a BPS state state in the theory and one expects to be able to localize it anywhere in the bulk supergravity geometry. The situation is therefore similar to that of D-instantons in AdS space [9] where the location of the D-instanton explicitly maps to the scale size of the Yang Mills instanton and one point functions in the D-instanton background accurately reproduce the Yang Mills instanton profile [10]. The hope is that in the non-commutative case as well the study of instantons for the self dual case would be revealing in the study of the IR/UV relation and more generally holographyt.

Another, and perhaps deeper, reason for being interested in the supergravity background for the case $B^{-}=0$ comes from attempts to understand holography in flat space. As we will see below, the Einstein frame metric for self dual $B$ field configurations, in the scaling limit which corresponds to noncommutative Yang Mills theory, is the full three brane geometry in the absence of a $B$ field. This remarkable fact has several implications. The most noteworthy being that the geometry with a self dual $B$ field is in fact asymptotically flat. We should add that other fields in particular the dilaton and axion are not constant. Even so, this fact suggests that ultraviolet noncommutative Yang Mills theory should help in understanding holography in flat 10-dim. space. It has been suggested in [12] that in the case without B fields the full D3 brane geometry corresponds to keeping the Born-Infeld action in the Yang Mills theory and not truncating to the lowest dimension operator. Here we see that the effect of exciting the other fields in supergravity- dilatonaxion and two forms- corresponds in the Yang Mills theory to a different choice of higher dimension operators -those which can be organised into the noncommutative Yang Mills Lagrangian.

This paper is structured as follows. In section 2, we review the supergravity solution for a self dual $B$ field and in particular show that its near horizon limit gives rise to the full three brane geometry without a B field turned on. In section 3, we then construct the Dinstanton solution. Because of the remarks above, this is also a solution for a D-instanton

4 Of course instantons in the general case when $B^{-} \neq 0$ are also interesting, perhaps even more so, since in that case the moduli space is different from the ordinary Yang Mills case and in particular the small instanton singularity is absent [11]. However, since these are not threshold bound states the supergravity solutions are harder to find. 
in the full D3 brane geometry without a B field. Section 4, discusses the supersymmetries of the solution, and finally section 5 , some aspects of holography.

\section{Supergravity Solutions with self dual B fields}

\subsection{The backgound geometry}

We will find euclidean solutions to the IIB supergravity equations of motion which are listed in Appendix I. In our notation, the NS fields will be real while the RR fields will be purely imaginary. Start with the following ansatz, for the dilaton $\phi$, RR scalar $\chi$, and NS and RR two form potentials $B_{\mu \nu}$ and $\tilde{B}_{\mu \nu}$.

$$
\begin{gathered}
\chi+i \gamma e^{-\phi}=i c \\
\tilde{H}_{\mu \nu \alpha}=i c H_{\mu \nu \alpha} \\
B_{01}=-\gamma B_{23} \quad \tilde{B}_{01}=-\gamma \tilde{B}_{23}
\end{gathered}
$$

where $c$ is a real constant. Here $H$ and $\tilde{H}$ denote the field strengths for $B$ and $\tilde{B}$. This ansatz is motivated by supersymmetry in the following section.

With this ansatz, the energy momentum tensors of $\phi$ and $\chi$ cancel each other and so does those of $B$ and $\tilde{B}$ so that Einstein equations (7.1) become

$$
R_{\mu \nu}-\frac{1}{2} g_{\mu \nu} R=T_{\mu \nu}^{F}
$$

where $T_{\mu \nu}^{F}$ denotes the stress energy of the five form field strength. The five form in turn is determined by Gauss' law

$$
\frac{1}{\sqrt{g}} \partial_{\mu}\left(\sqrt{g} F^{\mu \lambda \alpha \beta \gamma}\right)=0 .
$$

Finally, the dilaton and the axion equations $((7.2)$ and (7.3) respectively) both reduce to the following equation

$$
\nabla^{2}\left(e^{\phi}\right)+\frac{1}{6} H_{\mu \nu \alpha} H^{\mu \nu \alpha}=0
$$

while the $B$ and the $\tilde{B}$ equations ((7.4) and (7.5) respectively) both become

$$
\frac{1}{\sqrt{g}} \partial_{\mu}\left(\sqrt{g} H^{\mu \nu \lambda}\right)-\frac{2}{3} i F^{\nu \lambda \alpha \beta \gamma} H_{\alpha \beta \gamma}=0 .
$$

The important point is that with the ansatz, 2.2) (2.1) the equations for the metric (2.3) and the 5-form, (2.4) form a closed set independent of the two-forms and the dilaton 
and axion fields. Thus if a solution consistent with the ansatz (2.2) exists it give rise to a metric and 5-form identical to the case of the D3 brane solution without any two-form fields turned on.

One of the supergravity backgrounds presented in [6] is in fact a solution of the equations of motion with the ansatz (2.2). The metric (in the Einstein frame) is given 0 by

$d s_{E}^{2}=\frac{1}{\sqrt{g_{s}}}\left[\left[1+\frac{4 \pi g_{s} N\left(\alpha^{\prime}\right)^{2}}{r^{4}}\right]^{(-1 / 2)}\left(d x_{0}^{2}+\cdots+d x_{3}^{2}\right)+\left[1+\frac{4 \pi g_{s} N\left(\alpha^{\prime}\right)^{2}}{r^{4}}\right]^{(1 / 2)}\left(d r^{2}+r^{2} d \Omega_{5}^{2}\right)\right]$

The five form is

$$
F_{0123 r}=\left(\frac{4 i}{g_{s}}\right)\left[\frac{\left(4 \pi g_{s} N\right)^{2}\left(\alpha^{\prime}\right)^{2}}{\left(1+\frac{4 \pi g_{s} N\left(\alpha^{\prime}\right)^{2}}{r^{4}}\right)^{2}}\right] \frac{1}{r^{5}},
$$

and the dilaton, axion and two -forms are

$$
\begin{aligned}
e^{-\phi_{0}} & =\frac{\cos ^{2} \theta}{g_{s}}-i \chi_{0}=\frac{1}{g_{s} h(r)} \\
\tilde{B}_{01} & =\tilde{B}_{23}=-\frac{i \cos ^{2} \theta}{g_{s}} B_{01}=-\frac{i \cos ^{2} \theta}{g_{s}} B_{23}=-\frac{i}{g_{s}} \sin \theta \cos \theta \frac{h(r)}{f(r)}
\end{aligned}
$$

where

$$
\begin{aligned}
& f(r)=1+\frac{\alpha^{2} R^{4}}{r^{4}} \\
& h^{-1}(r)=\sin ^{2} \theta f^{-1}(r)+\cos ^{2} \theta
\end{aligned}
$$

The parameter $\theta$ is related to the $B$-fields at $r=\infty, g_{s}$ is the asymptotic value of the string coupling and

$$
R^{4}=\frac{4 \pi g_{s} N}{\cos ^{2} \theta}
$$

This is a solution of the equations of motion with the ansatz (2.2) with $c=-\frac{\cos ^{2} \theta}{g_{s}}$ and $\gamma=-1$. A similar solution can be of course written down with $\gamma=1$. In particular we note that the metric and five-form are independent of $\theta$ and equal to their values for a $D 3$ brane geometry without any $B$ fields turned on.

Now one can take a scaling limit which corresponds to the low-energy limit of [2] that gives rise to noncommutative Yang Mills theory. This is given by [6]:

$$
\alpha^{\prime} \rightarrow 0 \quad \theta \rightarrow \frac{\pi}{2}
$$

5 The solution below differs from that given in [6] by a relative negative sign between the RR and the NS-NS 2-form fields. We have checked explicitly that the following solves the equations of motion. 
with

$$
\tilde{b}=\alpha^{\prime} \tan \theta, \quad \tilde{g}=\frac{g_{s}}{\left(\alpha^{\prime}\right)^{2}} \text { and } r / \alpha^{\prime}
$$

held fixed.

The reader will notice that the harmonic function appearing in the metric cannot be not truncated to its near horizon value 6. Instead, as was mentioned in the introduction, the full D3 brane metric survives in this limit.

The full solution in this limit can be written in terms of the variable

$$
u=r /\left(\alpha^{\prime} R^{2}\right)
$$

used in [6]. After the rescaling coordinates $x_{i}$ along the brane as:

$$
x_{i} \rightarrow \tilde{x}_{i}=\frac{\tilde{b}}{\alpha^{\prime}} x_{i} \quad i=0,1,2,3
$$

the solution becomes

$$
\begin{aligned}
& d s^{2}=\frac{\alpha^{\prime} R^{2}}{\sqrt{\hat{g}}}\left[(f(u))^{-1 / 2}\left(d \tilde{x}_{0}^{2}+\cdots+d \tilde{x}_{3}^{2}\right)+(f(u))^{1 / 2}\left(d u^{2}+u^{2} d \Omega_{5}^{2}\right)\right] \\
& e^{-\phi_{0}}=\frac{1}{\hat{g}}-i \chi_{0}=\frac{1}{\hat{g}} u^{4} f(u) \\
& \tilde{B}_{01}=\tilde{B}_{23}=-\frac{i}{\hat{g}} B_{01}=-\frac{i}{\hat{g}} B_{23}=-\frac{i \alpha^{\prime} a^{2} R^{2}}{\hat{g}}(f(u))^{-1} \\
& F_{0123 u}=\frac{4 i\left(\alpha^{\prime}\right)^{2} R^{4}}{\hat{g} u^{5}}(f(u))^{-2}
\end{aligned}
$$

where

$$
f(u)=\frac{1}{u^{4}}+a^{4}
$$

and

$$
a^{2}=\tilde{b} R^{2}
$$

The coupling has been also redefined

$$
\hat{g}=\tilde{b}^{2} \tilde{g}
$$

while $R^{4}$, defined in (2.11) can be expressed as

$$
R^{4}=4 \pi \hat{g} N
$$

\footnotetext{
6 This is because the string coupling is also being scaled to zero.
} 
Note that the coupling $\hat{g}$ is precisely the open string coupling which appears in noncommutative Yang-Mills theory in the low energy limit defined in [2].

The metric (2.16) becomes $A d S_{5} \times S^{5}$ near the horizon, $u=0$ and $\hat{g}$ is the value of the string coupling in the IR. The $B$ fields vanish at $u=0$ and asymptote to $\alpha^{\prime} / \tilde{b}$ as $u \rightarrow \infty$.

It is important to note that the scaling limit considered above is not the near-horizon limit of the full geometry. This is the supergravity reflection of a corresponding feature of the low energy limit of the D3 brane theory considered in [2], viz. the fact that arbitrarily high powers of the (ordinary) field strengths survive.

In the rest of the paper we will work with the variables of (2.16)and rename $\tilde{x} \rightarrow x$.

\subsection{D-instanton Solution}

We now turn to determining the D-instanton solution in the background (2.7). We have already emphasized that (2.3) and (2.4) are independent of the two-forms and the dilaton-axion fields. In addition, the equation (2.6) for the three-form field strength does not involve the dilaton or axion fields either. Denoting the dilaton and axion fields appearing in (2.7) as $\phi_{0}, \chi_{0}$ it then follows that a solution different from (2.9) can be obtained with the same Einstein metric, two form fields and five form field strength but with different dilaton and axion values $\phi$ and $\chi$

$$
e^{\phi}=e^{\phi_{0}}+e^{\bar{\phi}} \quad \chi=\chi_{0}+\bar{\chi}
$$

so that

$$
i \chi+e^{-\phi}=\frac{1}{\hat{g}}
$$

and

$$
\nabla^{2}\left(e^{\bar{\phi}}\right)=0
$$

We are now ready to write down the equation satisfied by the fields due to a Dinstanton. The metric, $B$-fields and the five form field strength are exactly the same as in (2.9). Since the D-instanton couples to the dilaton and axion, the equation satsified by $\bar{\phi}$ is modified from (2.23) at the location of the instanton.

The D-instanton action is given by,

$$
S_{I}=2 \pi \int\left[e^{-\phi}+i \chi\right] \delta^{10}(x-\bar{x}) d^{10} x .
$$

Here $\bar{x}=\left(\bar{x}^{0} \cdots \bar{x}^{3}, \bar{u}, \bar{\theta}_{i}\right)$ is the location of the D-instanton (with $\theta_{i}$ denote the angles parametrizing the $S^{5}$ ). Thus the equation for the dilaton field is given by

$$
e^{\phi(x, \bar{x})}=e^{\phi_{0}(u)}+e^{\bar{\phi}(x, \bar{x})}
$$


where

$$
\nabla_{x}^{2}\left(e^{\bar{\phi}(x, \bar{x})}\right)=-4 \pi \kappa_{10}^{2} \frac{\delta^{10}(x-\bar{x})}{\sqrt{g}} .
$$

In other words we have to solve for the scalar Green's function in the metric given in (2.9).

This establishes the existence of the D-instanton solution in the presence of D3 branes and a nonzero B field. In the following section we will find explicit solutions to (2.26) in the decoupling limit, i.e in the background given by (2.16).

Before doing so let us make one comment regarding the instanton action (2.24). We started by an ansatz for solutions to supergravity equations. This ansatz can be in fact be motivated by the action (2.24). As was mentioned in the introduction, we expect in the self dual case that the three brane geometry should allow for a D instanton localised anywhere in the bulk. This means that the D-instanton action must be a constant. From (2.24) we see that this implies the condition (2.1). A similar argument then also shows that the full instanton and background solution satisfies (2.22). In the more general case where $B^{-} \neq 0$, and the instanton moduli space is deformed from the ordinary case, (2.24) will yield the potential generated in moduli space in the large 'tHooft coupling limit 7 .

\section{The smeared D-instanton solution}

We will now find the solution of 2.26) in the decoupling limit which is smeared over the $S^{5}$. In other words we look for solutions of the equation which do not depend on the angles $\theta_{i}$. This would give us the field due to a D-instanton which is smeared over the five sphere - the field would be then independent of $\theta_{i}$. Furthermore, using translation invariance along the brane directions $x^{0} \cdots x^{3}$ this solution may be written as

$$
e^{\bar{\phi}}=\frac{4 \kappa_{10}^{2} \hat{g}^{2}}{\pi^{2}\left(\alpha^{\prime} R^{2}\right)^{4}} \int d^{4} k \frac{1}{(2 \pi)^{4}} e^{-i k \cdot(x-\bar{x})} G_{k}(u, \bar{u}),
$$

where $G_{k}(u, \bar{u})$ now solves

$$
\partial_{u}\left(u^{5} \partial_{u} G_{k}\right)-u\left(1+a^{4} u^{4}\right) k^{2} G_{k}=-\delta(u-\bar{u})
$$

Note that we are looking for euclidean solutions so that $k^{2}>0$. Also $G_{k}$ should be regular as $u \rightarrow 0$, and $G_{k} \rightarrow 0$ when $u \rightarrow \infty$. The normalisation in (3.1) is obtained from (2.26) by integrating over the $S^{5}$ and noting that the volume of the unit five sphere is $\pi^{3}$.

7 We thank D. Tong for a conversation on this point. 
The Green's function can be expressed in terms of solutions to the homogeneous equation,

$$
\partial_{u}\left(u^{5} \partial_{u} G_{k}\right)-u\left(1+a^{4} u^{4}\right) k^{2} \psi_{k}=0
$$

by standard Strum-Liouville theory techniques. (3.3) was studied in [13] and subsequently also encountered in [6], for computing the two point function of a particular supergravity mode. Solutions to (3.3) can be written as

$$
\psi_{k}(\nu, u)=\frac{1}{u^{2}} \phi_{k}(\nu, u)
$$

where $\phi_{k}(\nu, w)$ solves the Mathieu equation,

$$
\left[\partial_{w}^{2}+2 q \cosh 2 w-4\right] \phi_{k}(\nu, w)=0
$$

with

$$
u=\frac{1}{a} e^{-w}
$$

and,

$$
q=-(k a)^{2}
$$

The parameter $\nu$ which enters the solution is determined by recursion relations and may be expressed as a power series in $(k a)$ as [13]

$$
\nu=2-\frac{i \sqrt{5}}{3}\left(\frac{\sqrt{q}}{2}\right)^{4}+\frac{7 i}{108 \sqrt{5}}\left(\frac{\sqrt{q}}{2}\right)^{8}+\cdots
$$

In order to implement the boundary conditions on the Greens function we need two solutions to (3.3) one being regular at $u \rightarrow 0$ and the second going to zero as $u \rightarrow \infty$. In the asymptotic region, $|w| \gg 1$, solutions to the Mathieu equation can be expressed in terms of modified Bessel functions. The two required solutions can then be written down as :

$$
\begin{aligned}
& \psi_{k}^{(1)}(\nu, u)=\frac{i \pi}{2 u^{2}} e^{\frac{i \pi \nu}{2}} H^{(1)}(\nu, w) \\
& \psi_{k}^{(2)}(\nu, u)=\frac{i \pi}{2 u^{2}} e^{\frac{i \pi \nu}{2}} H^{(1)}(\nu,-w)
\end{aligned}
$$

Here, we are using the notation of [13] and $H^{(1)}(\nu, w)$ denotes a solution which asymptotically is related to the modified Bessel function, $K_{\nu}$, i.e.,

$$
\frac{i \pi}{2} e^{\frac{i \pi \nu}{2}} H^{(1)}(\nu, w)=K_{\nu}\left(k a e^{w}\right) \rightarrow \sqrt{\frac{\pi}{2 k a e^{w}}} e^{-\left(k a e^{w}\right)} \quad w \gg 1
$$


- Thus we see that $\psi_{k}^{(1)}(\nu, u)$ is well behaved at $u=0$ while $\psi_{k}^{(2)}(\nu, u) \rightarrow 0$ at $u=\infty$, as required.

Now using Strum Liouville theory the Greens function is given by:

$$
\begin{aligned}
& G_{k}(u, \bar{u})=A^{2}(k, a) \psi_{k}^{(1)}(\nu, \bar{u}) \psi_{k}^{(2)}(\nu, u) \quad \bar{u}<u \\
& G_{k}(u, \bar{u})=A^{2}(k, a) \psi_{k}^{(1)}(\nu, u) \psi_{k}^{(2)}(\nu, \bar{u}) \quad \bar{u}>u
\end{aligned}
$$

The normalization $A^{2}(k, a)$ has to be determined by requiring that

$$
u^{5} W\left(\psi_{k}^{(1)}, \psi_{k}^{(2)}\right) A^{2}(k, a)=-1
$$

where $W\left(\psi_{k}^{(1)}, \psi_{k}^{(2)}\right)$ denotes the Wronskian of the two solutions. More detailed forms of the asymptotic behavior of these functions and the calculation of the Wronskian is given in Appendix II.

The result for the Wronskian fixes the normalization appearing in (3.11) to be

$$
A^{2}(k a)=\frac{2 \sin \pi \nu}{\pi}\left(\eta \zeta-\frac{1}{\eta \zeta}\right)^{-1}
$$

where $\zeta$ is defined in Appendix II.

We now have an expression for the solution to (2.26)

$$
e^{\bar{\phi}}=\frac{4 \kappa_{10}^{2} \hat{g}^{2}}{\pi^{2}\left(\alpha^{\prime} R^{2}\right)^{4}} \int\left[d^{4} k\right] \frac{1}{(2 \pi)^{4}} e^{-i k(x-\bar{x})} A^{2}(k a) \psi_{k}^{(1)}(\nu, \bar{u}) \psi_{k}^{(2)}(\nu, u) \quad u>\bar{u}
$$

The parameter $\nu$ and the function $\phi(\nu)$ (introduced in Appendix II) are explicitly defined in terms of the parameters in the Mathieu equation. However we will be able to extract some of the physics by knowing asymptotic forms. In Appendix II we derive the small $k a$ behavior of the various functions which appear in the solution to Mathieu's equation. In particular it is shown that

$$
A^{2}(k a) \sim(k a)^{4} \quad(k a)<<1
$$

Of particular interest is the small $(k a)$, fixed $(a u)$ behavior of the solutions. We reproduce the results from Appendix II

$$
\begin{aligned}
& \psi_{k}^{(1)}(\nu, u) \rightarrow \frac{1}{u^{2}} K_{2}(k / u) \\
& \psi_{k}^{(2)}(\nu, u) \rightarrow \frac{1}{u^{2}} K_{2}\left(k a^{2} u\right)
\end{aligned}
$$


This, together with (3.15) shows that the zero momentum Green's function is

$$
\begin{aligned}
\left.G_{k}(u, \bar{u})\right|_{k=0} & \sim \frac{1}{u^{4}} & & u>\bar{u} \\
\left.G_{k}(u, \bar{u})\right|_{k=0} & \sim \frac{1}{\bar{u}^{4}} & & u<\bar{u}
\end{aligned}
$$

This is what is expected from the differential equation definining $G_{k}$, equation (3.3) and is in fact the zero momentum Green's function in $A d S_{5}$ as well. Corrections to this extreme limit may be worked out systematically.

This shows that it is useful to recast (3.14) in a somewhat different form. The zero momentum component of $\psi_{k=0}^{(2)}(\nu, u)$ decays for large $u$ not exponentially but as a power law. It is useful for various purposes to show this dependence explicitly. Thus we can write:

$$
\begin{aligned}
e^{\bar{\phi}}=\frac{4 \kappa_{10}^{2} \hat{g}^{2}}{\pi^{2}\left(\alpha^{\prime} R^{2}\right)^{4}}\left[\frac{1}{4 L^{4} u^{4}}+\int\left[d^{4} k\right] \frac{1}{(2 \pi)^{4}} e^{-i k(x-\bar{x})} A^{2}(k, a) \psi_{k}^{(1)}(\nu, \bar{u}) \psi_{k}^{(2)}(\nu, u)\right] \\
u>\bar{u}
\end{aligned}
$$

where the first term is the zero momentum piece, with $L^{4}$ being the volume along the four directions parallel to the 3 branes. The integral in (3.18) is understood to be over the non-zero momentum modes.

It may be checked that the solution (3.18) reproduces known limits. When both $u$ and $\bar{u}$ are small and at small momenta we should recover the D-instanton in $A d S_{5} \times S^{5}$ of [9]. Similarly when both $u$ and $\bar{u}$ are large we should recover the D-instanton in flat space. This may be checked from the asymptotic expansions.

In the next section, we will discuss aspects of holography in this space-time. The boundary will be taken at $u=\infty$. The final results should reproduce the $A d S$ results in the $k a<<1$ limit. It is easy to see that in the limit $(k a)<<1$ with $y=k / u$ fixed the solutions reduce to the solutions for the $A d S_{5}$ problem. However the holographic correspondence we are interested in involves taking $u=\infty$ first, before taking the $(k a)<<1$ limit. This latter limit is nontrivial.

One comment is worth making at this point. We saw in section 2 that the D-instanton solution is given by the Greens function for $(2.26)$. The metric which appears in (2.26) is the full three brane metric. Thus (3.18) also provides the solution for the D-instanton in the full D3 brane geometry (with no two -form fields turned on). Although we will not do so here, using (2.14) (3.18) can be expressed in terms of the radial coordinate $r$ in a straightforward manner. 


\section{Supersymmetry of the solution}

The type IIB theory has thirty two supersymmetries. In this section we will consider a solution of the kind (2.1) (2.2) and show that it preserves sixteen of the thirty two supersymmetries. For definiteness we will consider the case where $\gamma=1$ which is the choice made in this paper. The supersymmetry variations of the dilatino and the gravitino are given by

$$
\begin{gathered}
\delta \lambda=\frac{-1}{2 \tau_{2}}\left(\frac{\tau^{*}-i}{\tau+i}\right) \Gamma^{\mu} \partial_{\mu} \tau\left(\eta_{1}-i \eta_{2}\right)-\frac{i}{24} \Gamma^{M N P}\left(\eta_{1}+i \eta_{2}\right) G_{M N P} \\
\delta \psi_{\mu}=\partial_{\mu}\left(\eta_{1}+i \eta_{2}\right)+\frac{1}{4} \omega_{\mu}^{a b} \Gamma^{a} \Gamma^{b}\left(\eta_{1}+i \eta_{2}\right)+\frac{i}{8 \tau_{2}}\left[\left(\frac{\tau-i}{\tau^{*}-i}\right) \partial_{\mu} \tau^{*}+\text { c.c. }\right]\left(\eta_{1}+i \eta_{2}\right) \\
+\frac{i}{480} \Gamma^{\mu_{1} \cdots \mu_{5}} \Gamma_{\mu} F_{\mu_{1} \cdots \mu_{5}}\left(\eta_{1}+i \eta_{2}\right) \\
-i \frac{1}{96}\left(\Gamma_{\mu}^{N P Q} G_{N P Q}-9 \Gamma^{N P} G_{M N P}\right)\left(\eta_{2}+i \eta_{1}\right)
\end{gathered}
$$

where

$$
\begin{gathered}
\tau=\tau_{1}+i \tau_{2}=\tau_{1}+i e^{-\phi} \\
G_{M N P}=i \sqrt{\tau_{2}}\left(\frac{|1-i \tau|}{\tau_{2}(1-i \tau)}\right)\left(H_{R R}-\tau H\right)_{M N P} .
\end{gathered}
$$

$\tau_{1}$ and $H_{R R}$ are related to the fields used in this paper by the relations:

$$
\begin{gathered}
\tau_{1}=-i \chi \\
H_{R R}=-i \tilde{H} .
\end{gathered}
$$

Thus, from (2.1) and $(\gamma=1)$ we have

$$
\tau_{2}-\tau_{1}=c
$$

and

$$
H_{R R}-\left(\tau_{2}-\tau_{1}\right) H=0 .
$$

(4.1) and (4.2) are supersymmetry transformations in Minkowski space. In going over to Euclidean space we follow [14] and replace the usual algebra of complex numbers generated over real numbers by $(1, i)$ with "hyperbolic" complex numbers generated over real numbers by $(1, e)$ with $e^{2}=1$. Thus $\left(\tau_{1}+i \tau_{2}\right) \rightarrow\left(\tau_{1}+e \tau_{2}\right), \eta=\eta_{1}+i \eta_{2} \rightarrow \eta_{1}+e \eta_{2}$ 
etc. Then using the fact that $e^{2}=1,(4.7)$ and $(4.8)$ it is easy to verify that the dilatino variation is zero if $\eta_{1}$ and $\eta_{2}$ satisfies the relation

$$
\eta_{1}=\eta_{2}
$$

As far as the gravitino variation is concerned we find from (4.7) (4.8) and (4.9) that the third term involving the derivative of $\tau$ and its complex conjugate vanish. Also the last term involving $G_{M N P}$ vanishes. Thus the gravitino variation becames:

$$
\delta \psi_{\mu}=\partial_{\mu}\left(\eta_{1}+e \eta_{2}\right)+\frac{1}{4} \omega_{\mu}^{a b} \Gamma^{a} \Gamma^{b}\left(\eta_{1}+e \eta_{2}\right)+\frac{e}{480} \Gamma^{\mu_{1} \cdots \mu_{5}} \Gamma_{\mu} F_{\mu_{1} \cdots \mu_{5}}\left(\eta_{1}+e \eta_{2}\right)
$$

We argued in section 2 that for the ansatz (2.1) (2.2) the metric and five form field strengths are independent of the two form fields and take the same form that they have in the D3 brane solution without any two-form expectation values. With this in mind we see that the (4.10) is exactly the same as for D3 branes without any additional two-form fields turned on. It follows then that the solution (2.1) (2.2) preserve sixteen supersymmetries. It also follows that the spinors $\eta_{1}, \eta_{2}$ which satisfy (4.10) and (4.9) are the same as in the case of D3 branes without the two-form.

\section{Holography}

D- instantons in $A d S_{5}$ were studied in [9] and shown to correspond to instantons in the Yang Mills theory. One expects the relationship to be more general and in particular to hold in the non-commutative Yang Mills theory as well. In this section we will study the correspondence further by calculating the action and correlation functions in the background of the D-instanton solution determined above and relating them to the corresponding quantities for an instanton in the Yang Mills theory. Our calculations will be different from the case of $A d S_{5}$ space in one important way. For the geometry under consideration here, the Einstein metric eq. (2.16) (to which the dilaton couples for example) asymptotically becames flat 10 dimensional spacetime. Thus in relating the D instanton to the Yang Mills instanton we will neccessarily have to deal with some of the difficulties of holography in flat space.

Below, we denote the supergravity solution described in Maldacena and Russo (with a antiself dual B field turned on ) as the background geometry. 
The $\mathrm{D}$ instanton solution in this background is given by:

$$
e^{\bar{\phi}}=C_{1} \int\left[d^{4} k\right] e^{-i k(x-\bar{x})} A^{2}(k, a) \psi_{k}^{(1)}(\nu, \bar{u}) \psi_{k}^{(2)}(\nu, u) \quad u>\bar{u}
$$

The axion is related to the dilaton through the condition

$$
e^{-\phi}+i \chi=\frac{1}{\hat{g}}
$$

and the metric and other three-form fields are the same as in the background solution.

To begin calculating in the supergravity theory we start with the supergravity action which is :

$$
S=\frac{1}{4 \kappa_{10}^{2}} \int d^{10} x \sqrt{g}\left[(\nabla \phi)^{2}+e^{2 \phi}(\nabla \chi)^{2}\right]+S_{B}+\cdots
$$

The bulk action is well known. $S_{B}$ above denotes a boundary term which is a little subtle to determine but important since it plays a crucial role in holography. For flat space the boundary term was determined in [15], by working in terms of a eight form gauge potential dual to the axion, to be:

$$
S_{B}=-\frac{1}{2 \kappa_{10}^{2}} \int d S^{n} e^{2 \phi} \chi \partial_{n} \chi
$$

15] showed that (5.4) gave the correct action for a D-instanton. One can also show that (5.4) gives the correct action for a D-instanton in AdS space. Carrying out a duality transformation to the eight form is not straightforward in the present context since the three form field strenghts etc. are turned on as well. Even so we will that the boundary term (5.4) is unchanged here as well 8 . As we will see below, this choice yields sensible answers at least for small perturbations about the background geometry.

\subsection{The instanton action}

One can now calculate the action for the instanton solution, (3.18). From (5.3) and (5.2) we see that the contribution from the bulk action vanishes. As was discussed in [15] since the D-instanton is a singular solution we need to consider two boundaries. One, as expected, is at large $u$, where the Einstein metric is asymptotically flat; the other is an infinitesimal surface surrounding the location of the D-instanton. Consider first the boundary at infinity. Since the background geometry is translationally invariant along the branes, only the zero momentum piece of the instanton solution (3.18) contributes to the boundary action. We denote the background as $\left(\phi_{0}, \chi_{0}\right)$ and write the dilaton

8 Intutively one would expect this boundary term because asymptotically the three form gauge potentials go to zero and one can at least in that region dualise the axion field. 
as $e^{\phi}=e^{\phi_{0}}+e^{\bar{\phi}}$ and $\chi=\chi_{0}+\chi_{I}$. Then using the fact that the full solution and the background satisfy (5.2) the leading dependence on the D-instanton in the boundary action (i.e. after subtracting an infinite contribution from the background) is :

$$
\Delta S_{B}=\frac{-i}{2 \kappa_{10}^{2}} \int d S^{n}\left(\chi_{0} \partial_{n} e^{\bar{\phi}}+\chi_{I} \partial_{n} e^{\phi_{0}}\right)
$$

Using the zero momentum piece from (3.18)and the condition (5.2) one finds that this vanishes. That leaves finally the second boundary: an infinitesimal surface around the location of the D-instanton. In this case one gets that the relevant term which contributes is :

$$
\Delta S_{B}=\frac{-i}{2 \kappa_{10}^{2}} \int d S^{n} \chi \partial_{n} e^{\bar{\phi}} .
$$

Since $e^{\bar{\phi}}$ satisfies the $(2.26)$ it diverges at the location of the D-instanton. Thus from (5.2) we see that $i \chi \rightarrow \frac{1}{\hat{g}}$. Substituting in (5.6) and using the equation satisfied by $e^{\bar{\phi}}$ (equation $(2.26))$, we finally get the action of the D-instanton to be

$$
S=\frac{2 \pi}{\hat{g}}
$$

This result agrees with the action of an instanton in the noncommutative Yang Mills theory. Note that, in the decoupling limit of [2] $\hat{g}$ is the open string coupling. This is related to the Yang Mills coupling by

$$
4 \pi \hat{g}=g_{Y M}^{2}
$$

(this can be shown for example from the Born Infeld action). Thus (5.7) yields,

$$
S=8 \pi^{2} / g_{Y M}^{2}
$$

This is in fact the classical action of an instanton in the non-commutative Yang Mills theory, as shown e.g. in [16] Further, since the instanton is a BPS state we expect its action to not get any quantum corrections and is therefore expected to agree with a supergravity computation.

It is worth pausing to emphasise that the calculation above in type II closed string theory yielded an answer in terms of the open string coupling constant $\hat{g}$. In fact $\hat{g}$ is related to the closed string coupling $g_{s}$ by the relation (2.19) involving the non-commutativity parameter explicitly. By way of contrast we note that had we not taken the scaling limit of the solution (2.9) an analysis along the above lines ( with the same boundary term 
) shows that the action of the D-instanton is $S=2 \pi / g_{s}$. The agreement between the D-instanton action (5.7) and the Yang Mills instanton obtained above provides additional evidence that the scaling limit (2.16) accurately implements the decoupling limit described in [2].

\subsection{One Point Functions}

As mentioned above, we would like to use the D instanton solution to calculate expectation values for operators in the instanton background of the Yang Mills theory. Three steps are involved determining the expecation values of operators in the Yang Mills theory from a supergravity solution. First, one determines the normal modes for small fluctuations about the background solution. Next, one computes the change in action to linear order in the small fluctuations. Finally, one determines the operator in the Yang Mills theory which corresponds to the supergravity normal mode and relates the change in the action to the one point function of the corresponding operator in the Yang Mills theory.

Here we will focus on one normal mode which satisfies the condition

$$
\delta \chi+i e^{-\phi} \delta \phi=0
$$

The discussion leading to the instanton solution in section 2 shows that a perturbation (5.10) satisfies the equations of motion if

$$
\nabla^{2}\left(e^{\phi} \delta \phi\right)=0
$$

Solutions to this equation behave asymptotically, for large $u$, like

$$
e^{\phi} \delta \phi \simeq \int \frac{d^{4} k}{(2 \pi)^{4}} C(\vec{k}) u^{-5 / 2}\left[e^{k a^{2} u}+B(k a) e^{-k a^{2} u}\right]
$$

In computing the one point function we first compute the change in the action for a perturbation about the background to second order. For this purpose it is useful to remember that when (5.10) is met, the full solution, consisting of background and perturbation, satisfies (5.2). Thus the bulk contribution vanishes and the change in the action arises entierly from the boundary term (5.4). Expanding this to second order gives 9 a surface term of the form:

$$
S=\frac{1}{2 \kappa_{10}^{2}} \int d S^{n} e^{-2 \phi_{0}}\left(e^{\phi} \Delta \phi\right) \partial_{n}\left(e^{\phi} \Delta \phi\right) .
$$

9 The one point function of this peturbation vanishes and this can be shown in exact analogy with the discussion of the instanton action above. 
The surface integral is at fixed value of $u=u_{0}$. Now we substitute

$$
\Delta \phi=\delta \phi+\phi_{I}
$$

where $\phi_{I}$ denotes the instanton background,

$$
e^{\phi_{0}}+e^{\bar{\phi}}=e^{\phi_{0}+\phi_{I}}
$$

and $\delta \phi$ is given by (5.12). This yields:

$$
S=\left(\frac{5 \pi}{u_{0}}\right) e^{-2 \phi_{0}} \int \frac{d^{4} k}{(2 \pi)^{4}} C(\vec{k}) A^{2}(k, a) \frac{1}{\sqrt{k a^{2}}} \psi_{k}^{(1)}(\nu, \bar{u})
$$

and a one-point function

$$
<O(\vec{k})>=\frac{\partial S}{\partial C(\vec{k})}=\left(\frac{5 \pi}{u_{0}}\right) e^{-2 \phi_{0}} \frac{A^{2}(k, a)}{(2 \pi)^{4}} \frac{1}{\sqrt{k a^{2}}} \psi_{k}^{(1)}(\nu, \bar{u})
$$

In order to relate this result to the gauge theory we need to decide what is the normalisation of the operator in the Yang Mills theory which couples to the perturbation. Unlike $A d S$ space, here there is considerable ambiguity in this matter. This issue was explored in [6] for a massless scalar field where it was found that a momentum dependent wave function renormalisation needed to be done to extract sensible answers. A similar renormalisation is required here as well 10. We have not been able to implement a satisfactory renormalisation scheme at the moment. The one which is most natural in the present context corresponds to a momentum dependent wave function renormalisation and therefore does not correpond to a choice of local counter terms. Below we will choose a renormalised operator whose one point function agrees at low momenta, and for a large instanton, with expectations from the ordinary gauge theory. The perturbation (5.10) should couple to the operator $\operatorname{Tr} F^{2}+\operatorname{Tr} F \tilde{F}$ at low momenta. Now the function $A^{2}(k, a)$ behaves for low momentum as:

$$
A^{2}(k, a)=c_{1}(k a)^{4}+\cdots
$$

Then consider the renormalised operator given by:

$$
O_{R}(\vec{k})=\frac{u_{0} \hat{g}}{5 c_{1}} \frac{e^{2 \phi_{0}}}{\left(k a^{2}\right)^{3 / 2}} O(\vec{k})
$$

10 This is clear if we consider a two point function of the perturbation above the background geometry. 
It's expectation value from (5.17) is

$$
<O_{R}(\vec{k})>=\frac{\pi}{\hat{g}} k^{2} \psi_{k}^{(1)}(\nu, \bar{u})
$$

When $a \bar{u}<<1$ the function $\psi_{k}^{(1)}(\nu, \bar{u}) \rightarrow \frac{1}{\bar{u}^{2}} K_{\nu}(k / \bar{u})$, and for $(k a)<<1$ we can replace $\nu \sim 2$. Then (5.20) reduces to exactly the answer for the one point function in the presence of a D-instanton in $A d S_{5} \times S^{5}$ 11. This is the Fourier transformation of

$$
\frac{1}{4 g_{Y M}^{2}}<\operatorname{Tr} F^{2}(x)+\operatorname{Tr} F \tilde{F}(x)>=\frac{48}{g_{Y M}^{2}} \frac{\left(\frac{1}{\bar{u}}\right)^{4}}{\left[\left(\frac{1}{\bar{u}}\right)^{2}+\left|\vec{x}-\vec{x}^{\prime}\right|\right]^{4}},
$$

which is the profile of an instanton in ordinary Yang-Mills theory.

Thus when the D-instanton is located deep in the bulk and we look at the long wavelength modes of the solution, the parameter $1 / \bar{u}$ in (5.20) plays the role of the instanton scale size as expected. It would be interesting to examine the departures from this low momentum behavior, as well as the predictions for the noncommutative Yang-Mills instantons of small size.

\subsection{Operators in the Gauge Theory}

In the usual $A d S-C F T$ correspondence, the dilaton $\phi$ couples to the operator as

$$
\int e^{-\phi} \operatorname{Tr}\left(F_{i j} F^{i j}\right)
$$

while the axion couples as

$$
\int \chi \operatorname{Tr}\left(F_{i j} \tilde{F}^{i j}\right)
$$

where $\tilde{F}$ stands for the dual in the four dimensional sense. One might think that to get the operators for noncommutative Yang-Mills theory one simply needs to replace ordinary gauge fields $F$ by nonocommutative gauge fields $\hat{F}$ and odrinary products by star products in the above relations. However this cannot be correct. Unlike in ordinary Yang -Mills theory, objects like $\operatorname{Tr} \hat{F} * \hat{F}$ are not gauge invariant though their integrals are 12 . As a result, while the operator $\operatorname{Tr} \hat{F}_{i j} * \hat{F}_{k l}$ can be coupled to supergravity fields which are constant along the brane (as for example in the form of the action for noncommutative Yang-Mills), one cannot couple them to nonconstant supergravity fields. However nontrivial effects of noncommutativity appear when there is momentum along the brane directions.

11 This may be verified by writing the D-instanton solution as a momentum space integral using the techniques of this paper and repeating the above calculation in that case.

12 S.T. acknowledges a discussion with A. Hashimoto on this issue. 
It is possible to write down gauge invariant operators order by order in the noncommutavity parameter and couple supergravity fields, but one suspects that there must be a more natural and simpler principle here. In a sense noncommutative gauge theories have a "nonabelian" nature when fields are regarded to have their momenta as group indices. Thus the natural invariant objects are in fact integrals over the brane rather than local quantities.

In any case, we believe that our supergravity results should act as a guide to the correct coupling of noncommutative gauge field with closed string fields.

\section{Acknowledgements}

We would like to thank Sunil Mukhi for discussions and collaboration at the early stages of this work and A. Hashimoto, M. Krogh, S. Mahapatra, P. Ramadevi and D. Tong for discussions. S.R.D would like to thank the participants of the Workshop on String Theory at MRI, Allahabad for discussions and questions which clarified many points. S.P.T. would like to acknowledge the hospitality of the ITP, Santa Barbara, where some of this work was completed as a participant in the workshop on Supersymmetric Gauge Theories, supported by the NSF grants, Phy 94-07194 and Phy 97-22022.

\section{Appendix I : Supergravity equations.}

The equations of motion for the bosonic fields in IIB supergravity in ten dimensions are, in terms of the einstein metric

$$
\begin{gathered}
R_{\mu \nu}-\frac{1}{2} g_{\mu \nu} R=T_{\mu \nu}^{\phi}+e^{-\phi} T_{\mu \nu}^{B}+e^{\phi} T_{\mu \nu}^{\tilde{B}}+e^{2 \phi} T_{\mu \nu}^{\chi}+T_{\mu \nu}^{F} \\
\frac{1}{\sqrt{g}} \partial_{\mu}\left(\sqrt{g} g^{\mu \nu} \partial_{\nu} \phi\right)+\frac{1}{12}\left[e^{-\phi} H^{2}-e^{\phi}(\tilde{H}-\chi H)^{2}\right]-e^{2 \phi} g^{\mu \nu} \partial_{\mu} \chi \partial_{\nu} \chi=0 \\
\frac{1}{\sqrt{g}} \partial_{\mu}\left(\sqrt{g} g^{\mu \nu} e^{2 \phi} \partial_{\nu} \chi\right)+\frac{1}{6}\left(\tilde{H}_{\mu \nu \lambda}-\chi H_{\mu \nu \lambda}\right) H^{\mu \nu \lambda}=0 \\
\frac{1}{\sqrt{g}} \partial_{\mu}\left(\sqrt{g} e^{-\phi} H^{\mu \nu \lambda}\right)-\frac{1}{\sqrt{g}} \partial_{\mu}\left(\sqrt{g} e^{\phi} \chi\left(\tilde{H}^{\mu \nu \lambda}-\chi H^{\mu \nu \lambda}\right)\right)+\frac{2}{3} F^{\nu \lambda \alpha \beta \gamma} \tilde{H}_{\alpha \beta \gamma}=0 \\
\frac{1}{\sqrt{g}} \partial_{\mu}\left(\sqrt{g} e^{-\phi}\left(\tilde{H}^{\mu \nu \lambda}-\chi H^{\mu \nu \lambda}\right)\right)-\frac{2}{3} F^{\nu \lambda \alpha \beta \gamma} H_{\alpha \beta \gamma}=0 \\
\frac{1}{\sqrt{g}} \partial_{\mu}\left(\sqrt{g} F^{\mu \lambda \alpha \beta \gamma}\right)=0
\end{gathered}
$$


The fields are as following. $\phi, \chi$ denote the dilaton and the axion. $B, \tilde{B}$ denote the NS and RR 2-form fields and $H, \tilde{H}$ are the corresponding field strengths which are defined as

$$
H_{\mu \nu \alpha}=\partial_{\mu} B_{\nu \alpha}+\partial_{\nu} B_{\alpha \mu}+\partial_{\alpha} B_{\mu \nu}
$$

and similarly for $\tilde{H}$ in terms of $\tilde{B} . F^{\nu \lambda \alpha \beta \gamma}$ is the self dual 5 -form field strength. The energy momentum tensors appearing in (7.1) are given by

$$
\begin{aligned}
T_{\mu \nu}^{\phi} & =\frac{1}{2}\left[\partial_{\mu} \phi \partial_{\nu} \phi-\frac{1}{2} g_{\mu \nu}(\partial \phi)^{2}\right] \\
T_{\mu \nu}^{\chi} & =\frac{1}{2}\left[\partial_{\mu} \chi \partial_{\nu} \chi-\frac{1}{2} g_{\mu \nu}(\partial \chi)^{2}\right] \\
T_{\mu \nu}^{B} & =\frac{1}{12}\left[3 H_{\mu \alpha \beta} H_{\nu}^{\alpha \beta}-\frac{1}{2} g_{\mu \nu} H^{2}\right] \\
T_{\mu \nu}^{\tilde{B}} & =\frac{1}{12}\left[3(\tilde{H}-\chi H)_{\mu \alpha \beta}(\tilde{H}-\chi H)_{\nu}^{\alpha \beta}-\frac{1}{2} g_{\mu \nu}(\tilde{H}-\chi H)^{2}\right] \\
T_{\mu \nu}^{F} & =\frac{1}{480}\left[5 F_{\mu \alpha_{1} \alpha_{2} \alpha_{3} \alpha_{4}} F_{\nu}^{\alpha_{1} \alpha_{2} \alpha_{3} \alpha_{4}}-\frac{1}{2} g_{\mu \nu} F^{2}\right]
\end{aligned}
$$

\section{Appendix II: Asymptotic forms and the Wronskian}

In this appendix we give the necessary asymptotic forms of the solutions and various quantities related to these solutions and compute the Wronskian necessary for normalization of the Green's function.

\subsection{Asymptotic forms}

The asymptotics of $\psi_{k}^{(i)}$ may be figured out by using the relation [13],

$$
H^{(1)}(\nu, w)=\frac{1}{2 i \sin \pi \nu}\left[\left(\zeta-\frac{1}{\zeta}\right) H^{(1)}(\nu,-w)+\left(\zeta-\frac{e^{-2 \pi i \nu}}{\zeta}\right) H^{(1)}(\nu,-w)\right]
$$

where we have defined

$$
\zeta=\frac{\phi(-\nu / 2)}{\phi(\nu / 2)}
$$

We will also define

$$
\eta=e^{i \pi \nu}
$$

$\phi(\nu)$ is the function of $\nu$ which appears in the Floquet form of the solution to Mathieu equation. The relation (8.1) yields the following asymptotics for $w \rightarrow-\infty(u \rightarrow \infty)$

$$
\begin{aligned}
\psi_{k}^{(1)}(\nu, u) & \rightarrow \frac{1}{u^{2}}\left[\frac{i e^{-i \pi \nu}}{\zeta} K_{\nu}\left(k a^{2} u\right)+\frac{\pi}{2 \pi \sin \pi \nu}\left(\zeta e^{i \pi \nu}-\frac{e^{-i \pi \nu}}{\zeta}\right) I_{\nu}\left(k a^{2} u\right)\right] \\
\psi_{k}^{(2)}(\nu, u) & \rightarrow \frac{1}{u^{2}} K_{\nu}\left(k a^{2} u\right)
\end{aligned}
$$


and similarly for $w \rightarrow \infty(u \rightarrow 0)$

$$
\begin{aligned}
& \psi_{k}^{(1)}(\nu, u) \rightarrow \frac{1}{u^{2}} K_{\nu}\left(\frac{k}{u}\right) \\
& \psi^{(2)}(\nu, u) \rightarrow \frac{1}{u^{2}}\left[\frac{i e^{-i \pi \nu}}{\zeta} K_{\nu}\left(\frac{k}{u}\right)+\frac{\pi}{2 \pi \sin \pi \nu}\left(\zeta e^{i \pi \nu}-\frac{e^{-i \pi \nu}}{\zeta}\right) I_{\nu}\left(\frac{k}{u}\right)\right]
\end{aligned}
$$

\subsection{The Wronskian}

It follows from standard Strum-Liouville theory that $W\left(\psi_{k}^{(1)}, \psi_{k}^{(2)}\right) u^{5}$ is a constant independent of $u$, so we can evaluate it for any $u$. In particular we may evaluate this for $w \rightarrow-\infty$.

The Wronskian can be now easily evaluated, giving

$$
W\left(\psi_{k}^{(1)}, \psi_{k}^{(2)}\right)=\frac{1}{u^{5}}\left[\frac{\pi}{2 \sin \pi \nu}\left(\eta \zeta-\frac{1}{\eta \zeta}\right)\right]
$$

This fixes the function $A^{2}(k a)$ defined in the text to be

$$
A^{2}(k a)=\frac{2 \sin \pi \nu}{\pi}\left(\eta \zeta-\frac{1}{\eta \zeta}\right)^{-1}
$$

\subsection{Power series expansions of various quantities}

The results of [13 may be used to obtain expressions for the various quantities defined above. First note that in our case, the expansion for $\nu$ ensures that $\eta$ is real. Then, as shown in [13], the quantity $\zeta$ has to have unit modulus. Let us denote

$$
\eta=e^{\beta} \quad \zeta=e^{i \alpha} \quad(\alpha, \beta \text { real })
$$

The quantity $\beta$ can be read off from the definition of $\nu$.

Define the quantities

$$
\begin{aligned}
& E=\zeta-\frac{1}{\zeta}=2 \sin \alpha \\
& F=\zeta \eta-\frac{1}{\zeta \eta}=2(\cos \alpha \sinh \beta+i \sin \alpha \cosh \beta) \\
& G=\eta-\frac{1}{\eta}=2 \sinh \beta
\end{aligned}
$$

The unitarity relation of the quantum mechanical problem corresponding to our basic differential equation

$$
|F|^{2}=|E|^{2}+|G|^{2}
$$


is now automatically satisfied.

In terms of the quantity

$$
P=\left|\frac{G}{F}\right|^{2}
$$

it is straightforward to show that

$$
\sin ^{2} \alpha=\sinh ^{2} \beta\left(\frac{1}{P}-1\right)
$$

We can now obtain expressions for $E, F, G$ in terms of $\beta$ and $P$. $\beta$ has been already given in the expansion for $\nu$ above,

$$
\beta=\frac{\sqrt{5}}{3}\left(\frac{k a}{2}\right)^{4}-\frac{7}{108 \sqrt{5}}\left(\frac{k a}{2}\right)^{8}+\cdots
$$

while $P$ has been expressed as a power series in [13]. In our notation this is

$$
P=\pi^{2}\left(\frac{k a}{2}\right)^{8} \sum_{n=0}^{\infty} b_{n, k}(k a)^{4 n}(\log (k a))^{k}
$$

where $b_{n, k}$ are some real coefficients tabulated in [13].

Of particular interest is the small $k a$ behavior of these quantities. These are

$$
\begin{aligned}
& E=e_{1}+O\left((k a)^{4}\right) \\
& F=i f_{1}+O\left((k a)^{4}\right) \\
& G=g_{a}(k a)^{4}+O\left((k a)^{8}\right)
\end{aligned}
$$

where $e_{1}, f_{1}, g_{1}$ are real numerical coefficients of order one.

$$
A^{2}(k a)=\frac{1}{i \pi} \frac{G}{F} \sim(k a)^{4}
$$

We will use these results to examine the low momentum components of the instanton solution.

\subsection{Low energy limit of solutions}

In the limit $(k a)<<1$ at fixed $(a u)$ the solutions $\psi_{k}$ go over to Bessel functions as well. This may be seen from the relation, valid for any $w$

$$
H^{(1)}(\nu, w)=\sum_{n=-\infty}^{\infty} \frac{\phi\left(n+\frac{n u}{2}\right)}{\phi\left(\frac{\nu}{2}\right)} J_{n}\left(i k a e^{-w}\right) H_{n+\nu}^{(1)}\left(i k a e^{w}\right)
$$

For $(k a)<<1$ with fixed $w$ only the $n=0$ term in the sum contributes, and we can also set $\nu=2$ in the leading order. We finally have, in this limit

$$
\begin{aligned}
\psi_{k}^{(1)}(\nu, u) & \rightarrow \frac{1}{u^{2}} K_{2}(k / u) \\
\psi_{k}^{(2)}(\nu, u) & \rightarrow \frac{1}{u^{2}} K_{2}\left(k a^{2} u\right)
\end{aligned}
$$




\section{References}

[1] A. Connes, M. Douglas and A. Schwarz, JHEP 9802 (1998) 003, hep-th/9711162; M. Douglas and C. Hull, JHEP 02 (1998) 008, hep-th/9711165.

[2] N. Seiberg and E. Witten, JHEP 9909:032 (1999), hep-th/9908142.

[3] Y.K.E. Cheung and M. Krogh, Nucl. Phys. B528 (1998) 185, hep-th/9803031; C.S. Chu and P.M. Ho, Nucl. Phys. B550 (1999) 151, hep-th/9812219; F. Ardalan, H. Arfaei and M.M. Sheik-Jabbari, JHEP 02:016 (1999), hep-th/9810072; V. Schomerus, JHEP 9906:030 (1999), hep-th/9903205; D. Bigatti and L. Susskind, hep-th/9908056.

[4] J. Maldacena, Adv.Theor. Math. Phys. 2 (1998) 231, hep-th/9711200; S. Gubser, I. Klebanov and A. Polyakov, Phys. Lett. B428 (1998) 105,hep-th/9802109; E. Witten, Adv. Theor. Math. Phys. 2 (1998) 253, hep-th/9802150

[5] A. Hashimoto and N. Itzhaki, hep-th/9907166.

[6] J. Maldacena and J. Russo, hep-th/9908134.

[7] M. Li and Y.S. Wu, hep-th/9909085.

[8] M. Alishahiha, Y. Oz and M. Sheikh-Jabbari, hep-th/9909215; A. Mikhailov, hepth/9910126; A. Dhar, G. Mandal, S.R. Wadia and K.P. Yogendran, hep-th/9910154.

[9] C.S. Chu, P.M. Ho and Y.Y. Wu, hep-th/9806103; M. Bianchi, M. Green, S. Kovacs and G. Rossi, hep-th/9807033

[10] V. Balasubramanium, P. Kraus, A. Lawrence and S.P. Trivedi, hep-th/9808017.

[11] N. Nekrasov and A. Schwarz, Comm. Math. Phys. 198 (1998) 689, hep-th/9802068

[12] S. Gubser, A. Hashimoto and I. Klebanov, Nucl. Phys. B 526 (1998) 393, hepth/9803023.

[13] S. Gubser and A. Hashimoto, Comm. Math. Phys. 203 (1999) 325, hep-th/9805140

[14] G. W. Gibbons, M. B. Green and M. Perry, Phys. Lett. B370 (1996), 37

[15] M. Green and M. Gutperle, hep-th/9701093

[16] A. Astashkevich, N. Nekrasov and A. Schwarz, hep-th/9810147. 